\title{
Short answer in short segment Barrett's oesophagus
}

Adenocarcinomas of the oesophagus and cardia share epidemiological and pathological features. Both are increasing at an alarming rate, are commoner in white men, show similar staining patterns for mucins, and are associated with specialised intestinal metaplasia. This has lead to the suggestion that these cancers represent the same disease at different locations.

The realisation that specialised intestinal metaplasia in the oesophagus is acquired consequent to gastro-oesophageal reflux disease (GORD) and that it is the initiating factor in a metaplasia to dysplasia to adenocarcinoma sequence suggests that this lethal cancer can be prevented by effective treatment of reflux disease. Endoscopic screening of the oesophagus in patients with specialised intestinal metaplasia is now widely practiced so that dysplasia and adenocarcinoma may be detected at an early, curable stage. The benefit of this approach is becoming apparent as surgical series of patients with dysplasia and adenocarcinoma detected via screening show improved survival, with low morbidity. It remains to be determined whether patients with specialised intestinal metaplasia at or below the gastro-oesophageal junction should be offered surveillance.

Histological examination of the epithelium of the gastrooesophageal junction has been neglected until recently. This is surprising in view of the notable similarities between adenocarcinomas of the cardia and the oesophagus, and the high prevalence of GORD in the West. Changes in this epithelium are probably linked to both of these conditions. An unexpected finding in the reports to date is the high prevalence of unsuspected specialised intestinal metaplasia at the cardia in patients undergoing endoscopy for a variety of reasons. Although adenocarcinoma of the cardia is more common than oesophageal adenocarcinoma, the high rate of specialised intestinal metaplasia in the cardia suggests that the risk of malignant degeneration is smaller than for this epithelium lining the oesophagus. The risk has not been quantified yet.

There is an inherent difficulty in saying that there is a risk, warranting surveillance of patients with specialised intestinal epithelium in the oesophagus and not for patients with this epithelium at or below the gastro-oesophageal junction as it is difficult to be sure, endoscopically, where the oesophagus ends. Thus, when a biopsy sample is taken from the gastro-oesophageal junction it is hard to know whether the sample is from the oesophagus or not. A potentially useful and practical classification for biopsy site is: below the gastro-oesophageal junction (below the gastric rugal folds), at the gastro-oesophageal junction (within $2 \mathrm{~cm}$ above the gastric rugal folds), above the gastro-oesophageal junction (proximal to $2 \mathrm{~cm}$ above the gastric rugal folds), and indeterminate when the upper extent of the gastric rugal folds is indistinct. This will permit quantification of the risk of subsequent malignant degeneration for patients with specialised intestinal metaplasia at these locations. Some consensus is required so that reports from different authors may be compared.

The paper by Nandurkar et al (see page 710) shows that the prevalence of specialised intestinal metaplasia in the region of the gastro-oesophageal junction (in patients without $3 \mathrm{~cm}$ of recognisable circumferential columnar lining above the gastro-oesophageal junction) is $15 \%$ on routine haematoxylin and eosin stained sections and $30 \%$ on sections stained with alcian blue. The high prevalence is in agreement with previous reports and confirms the superiority of alcian blue in detecting goblet cells. Of note, specialised intestinal metaplasia below the squamocolumnar junction is more prevalent in older subjects and is associated with microscopic, if not macroscopic, inflammation in the oesophagus. This suggests a link between GORD and specialised intestinal metaplasia at this location. Microscopic evidence of "oesophagitis" may be caused by GORD and, if so, is evidence of increased contact between the oesophageal mucosa and refluxate from the stomach into the oesophagus. It is not as sensitive or specific as ambulatory $\mathrm{pH}$ metry. The association between microscopic oesophagitis and specialised intestinal metaplasia suggests that injury to the gastro-oesophageal junction is a part of reflux disease. This offers the intriguing possibility that damage to the epithelium in the region of the cardia is an early event in the pathogenesis of reflux disease.

Further research into this area is needed and no recommendation can be made as yet, whether surveillance is indicated for patients with specialised intestinal metaplasia at or below the gastro-oesophageal junction. Consensus is needed on classification of the site of specialised intestinal metaplasia. Study of the association between injury to the epithelium in this region and reflux disease should provide further information on the pathogenesis of reflux disease and of adenocarcinomas of the cardia and oesophagus.

ADRIAN IRELAND

Department of Surgery,

USC School of Medicine,

1510 San Pablo Street, Suite 514

Los Angeles, CA 90033-4612, USA

1 Clark GWB, Ireland AP, Peters JH, Chandrasoma P, DeMeester TR, Bremner CG. Short segment Barrett's esophagus: A prevalent
complication of gastroesophageal reflux disease with malignant potential. complication of gastroesophageal reflux

2 Clark GWB, Ireland AP, Chandrasoma P, DeMeester TR, Peters JH, Bremner CG. Inflammation and metaplasia in the transitional epithelium of the gastroesophageal junction: a new marker for gastroesophageal reflux disease [abstract]. Gastroenterology 1994; 106: A63 